\title{
The $k_{\perp}$-Clustering Algorithm for Jets in Deep Inelastic Scattering and Hadron Collisions*
}

\author{
S. Catani ${ }^{\dagger}$ \\ Theory Division, CERN, CH-1211 Geneva 23, Switzerland \\ Yu.L. Dokshitzer \\ Nuclear Physics Institute, Gatchina, St. Petersburg 188350, Russia and \\ Department of Theoretical Physics, University of Lund, \\ Sölvegatan 14A, S-22362 Lund, Sweden \\ and \\ B.R. Webber ${ }^{\ddagger}$ \\ Theory Division, CERN, CH-1211 Geneva 23, Switzerland
}

\begin{abstract}
We propose a new QCD-motivated clustering algorithm to define jets in leptonhadron and hadron-hadron collisions. It combines the $k_{\perp}$-algorithm, proposed earlier for $e^{+} e^{-}$annihilation, with a pre-clustering procedure that ensures the universal factorization of initial-state collinear singularities.
\end{abstract}

CERN-TH.6473/92

LU TP 92-14

March 1992

\footnotetext{
* Research supported in part by the Italian Ministero dell'Università e Ricerca Scientifica.

${ }^{\dagger}$ On leave of absence from INFN, Sezione di Firenze, Italy.

$\ddagger$ On leave of absence from Cavendish Laboratory, University of Cambridge, UK.
} 



\section{Introduction}

A jet can be defined as a large amount of hadronic energy in a small angular region. According to this qualitative definition, the first Evidence for Jet Structure in Hadron Production by $e^{+} e^{-}$Annihilation was reported in 1975 [1]. Since then, jet production in $e^{+} e^{-}$and hadron colliders has become one of the main tools for investigating strong interaction physics and testing QCD.

We are nowadays in a position to use jet cross section data for both

- precise quantitative tests of QCD (measurement of the QCD coupling $\alpha_{S}$ and scale $\Lambda_{\overline{\mathrm{MS}}}$, study of QCD coherence), and

- looking for breakdown of the standard model and new physics,

provided the qualitative definition of a jet given above is replaced by a precise quantitative definition. We need a jet algorithm able to specify a jet configuration unambiguously starting from the hadrons detected in the final state.

\subsection{Jets in $e^{+} e^{-}$annihilation}

The problem of defining a jet algorithm for $e^{+} e^{-}$-annihilation has been reconsidered recently [2-7]. Let us briefly review the main developments before setting up our proposal for collisions involving hadrons.

For $e^{+} e^{-}$annihilation the jet definition has to fulfil the requirements of being

i) infrared (IR) and collinear safe

ii) simple to use in experimental analyses

iii) simple to use in theoretical calculations

iv) subject to small hadronization corrections.

These requirements are implemented in a jet algorithm defining

$\left.j_{1}\right)$ a test variable (energy-angle resolution)

$\left.j_{2}\right)$ a recombination procedure.

The test variable is needed in order to specify whether or not two final state hadrons belong to the same jet, whilst the recombination procedure tells us how the properties of a jet are related to those of the hadrons belonging to it.

At present there are essentially two classes of jet algorithms in use [8]: clustering algorithms of the type firstly introduced by the J ADE collaboration [9] and cone-type algorithms 'à la Sterman-Weinberg' [10]. In some respects, clustering algorithms are more precisely defined because they do not suffer from ambiguities related to dealing with overlapping cones in multijet events. It is surely true that clustering algorithms are more suitable for $e^{+} e^{-}$-annihilation. 
Several clustering algorithms for $e^{+} e^{-}$collisions have been examined. In our opinion [2-4] the requirements i) - iv) above are maximized by the $k_{\perp}$-algorith ${ }^{\S}$ whose definition is as follows.

Let us consider an $n$-hadron final state produced by $e^{+} e^{-}$annihilation

$$
e^{+}\left(p_{+}\right)+e^{-}\left(p_{-}\right) \rightarrow h_{1}\left(p_{1}\right)+\cdots+h_{n}\left(p_{n}\right), \quad p_{+}+p_{-} \equiv Q .
$$

We denote by $E_{i}$ and $\theta_{i j}$ respectively energies and angles in the centre-of-mass frame of the incoming leptons. The $k_{\perp}$-algorithm is defined according to the following iterative procedure.

1) Define a resolution parameter $y_{\text {cut }}$.

2) For every pair of hadrons $h_{k}, h_{l}$ compute the corresponding relative transverse momentum

$$
y_{k l}=\frac{2\left(1-\cos \theta_{k l}\right)}{Q^{2}} \min \left(E_{k}^{2}, E_{l}^{2}\right) .
$$

3) If $y_{i j}$ is the smallest value of $y_{k l}$ computed in 2) and $y_{k l}<y_{\text {cut }}$, combine $\left(p_{i}, p_{j}\right)$ into a single jet ('pseudoparticle') $p_{i j}$ according to a recombination prescription.

4) Repeat this procedure from step 2) until all pairs of objects (particles and/or pseudoparticles) have $y_{k l}>y_{\text {cut }}$. Whatever objects remain at this stage are called jets.

According to the classification given by $\left.\left.j_{1}\right), j_{2}\right)$ in Sec. 1 , the $k_{\perp}$-algorithm uses the scaled transverse momentum (2) as test variable. In the experimental and theoretical analyses several different recombination procedures may be used. They differ among themselves in the way in which the the 4 -momentum $p_{i j}$ of a pseudoparticle is defined in terms of the momenta $\left(p_{i}, p_{j}\right)$ of the recombined particles and/or pseudoparticles. The simplest recombination prescription is the so called E-scheme, in which

$$
p_{i j}=p_{i}+p_{j}
$$

For historical reasons other recombination schemes have been considered. The point is that it was noticed that in the original JADE algorithm [9] (in which the invariant mass is used as test variable) the jet properties and in particular the size of hadronization corrections were strongly affected by the recombination procedure. In the case of the $k_{\perp}$-algorithm that is no longer true. Therefore, when considering hadron collisions in the following Sections we assume that the E-scheme recombination (3) is used (although other recombination prescriptions could be defined as well).

\subsection{Jets in hadron collisions}

In the case of collisions involving hadrons there are new features with respect to $e^{+} e^{-}$ annihilation. In deep inelastic lepton-hadron scattering (DIS), for instance, one has to deal with the proton remnant and isolate the latter from high- $p_{\perp}$ jets produced not only in the current fragmentation region but also in the target fragmentation region as well. I

\footnotetext{
$\S_{\text {The }} k_{\perp}$-algorithm for $e^{+} e^{-}$annihilation is also known as the Durham algorithm. It is a subset of the $k_{\perp}$-algorithm for all types of collisions, to be defined in Secs. 2 and 3.

Other approaches to these problems are studied in Ref. [11].
} 
In hadron-hadron collisions the centre-of-mass energy does not control the hardness of the process: the high- $p_{\perp}$ scattering process has to be properly defined and separated from the underlying soft event. Moreover, from the point of view of perturbative QCD, initial state collinear singularities have to be factorized in a universal (process independent) way in order for the hard scattering part to be computable in perturbation theory. Therefore jet algorithms for hadron collisions have to fulfil extra requirements with respect to those needed for $e^{+} e^{-}$annihilation. We add to the previous list i) - iv) the requirements of

v) allowing the universal factorization of initial state collinear singularities

vi) minimizing contamination from the hadron remnant and underlying event.

In the definition of the algorithm not only the jet resolution and the recombination procedure $\left.j_{1}\right), j_{2}$ ) have to be considered but we also have to introduce

$\left.j_{3}\right)$ a hard scattering scale.

In Sec. 2 we define a new jet algorithm for DIS and we also consider the photoproduction limit. Sec. 3 is devoted to proposing a similar algorithm for hadron-hadron collisions. Some results of QCD calculations using these new algorithms are reported in Sec. 4. Finally, our main results are summarized and discussed in Sec. 5.

\section{The $k_{\perp}$-algorithm for DIS}

\subsection{Definition}

In this section we propose a new clustering algorithm for jets produced in the deep inelastic lepton-hadron scattering process

$$
\ell\left(p_{\ell}\right)+h(p) \rightarrow \ell^{\prime}\left(p_{\ell}^{\prime}\right)+h_{1}\left(p_{1}\right)+\cdots h_{n}\left(p_{n}\right) .
$$

Here $h(p), \ell\left(p_{\ell}\right)$ and $\ell^{\prime}\left(p_{\ell}^{\prime}\right)$ are the initial state hadron, the initial state lepton and the final state lepton respectively. DIS cross sections are usually expressed in terms of the invariants $x, Q^{2}$ and $y$

$$
\begin{aligned}
q=p_{\ell}-p_{\ell}^{\prime}, & Q^{2}=-q^{2} \\
x=\frac{Q^{2}}{2 p q}, & y=\frac{p q}{p p_{\ell}} .
\end{aligned}
$$

The $k_{\perp}$-algorithm is defined in the Breit frame of the initial state hadron and the exchanged vector boson

$$
p=E(1, \mathbf{0}, 1), q=(0, \mathbf{0},-2 x E), Q^{2}=4 x^{2} E^{2} .
$$

We denote by $E_{i}$ the energy of the final-state hadron $h_{i}$, by $\theta_{i j}$ the angle between the momenta of hadrons $h_{i}$ and $h_{j}$, and by $\theta_{i p}$ the angle of the hadron momentum $p_{i}$ with respect to the initial state hadron direction, all in the Breit frame (6). Jets are defined by a two-step procedure which amounts to A) pre-clustering of hadrons into a 'beam jet' and 'final-state macro-jets' and B) resolving final-state jets within the macro-jets. 
A) Pre-clustering of hadrons.

$\left.a_{1}\right)$ Define a hard scattering scale $E_{t}\left(Q^{2} \geq E_{t}^{2} \gg \Lambda^{2}, \Lambda\right.$ being the QCD scale $)$

$a_{2}$ ) For every final state hadron $h_{k}$ compute

$$
y_{k p}=\frac{2\left(1-\cos \theta_{k p}\right)}{E_{t}^{2}} E_{k}^{2},
$$

as well as for every pair $h_{k}, h_{l}$,

$$
y_{k l}=\frac{2\left(1-\cos \theta_{k l}\right)}{E_{t}^{2}} \min \left(E_{k}^{2}, E_{l}^{2}\right) .
$$

$\left.a_{3}\right)$ Take the smallest value among $\left\{y_{k l}, y_{k p}\right\}$. If $y_{i j}$ is the smallest value and $y_{i j}<1$, combine $\left(p_{i}, p_{j}\right)$ into a single pre-cluster ('pseudoparticle') $p_{i j}=p_{i}+p_{j}$. If $y_{i p}<1$ is the smallest value, include $p_{i}$ in the 'beam jet'.

$a_{4}$ ) Repeat this procedure from step $a_{2}$ ) for all particles (pseudoparticles) not included in the beam jet, until all objects (particles and/or pseudoparticles) have $y_{k l}, y_{k p}>1$. One ends up with the beam jet and objects which we call final-state macro-jets.

B) Resolving jet structure.

$b_{1}$ ) Define a resolution parameter $y_{\text {cut }}=Q_{0}^{2} / E_{t}^{2}<1$.

$\left.b_{2}\right)$ For any hadron in a final-state macro-jet consider $y_{i j}$ and define final-state jets as for $e^{+} e^{-}$annihilation in Sec. 1.1.

The results of the full procedure are jets with relative transverse momenta $\Delta k_{\perp}$ where $E_{t}>\Delta k_{\perp} \geq Q_{0}=\sqrt{y_{\text {cut }}} E_{t}$, produced within macro-jets with large transverse momenta $p_{\perp} \geq E_{t}$ relative to each other and the incoming hadron.

The above definition provides a QCD-motivated implementation of the jet requirements i) - vi). The two-step procedure allows us to include the proton remnants in the beam jet while associating with jets the coherent (small-relative-angle) soft radiation accompanying the hard parton scattering.

In experimental as well as theoretical analyses one can use any hard scattering scale $E_{t}^{2} \leq Q^{2}$. For fixed values of $y_{\text {cut }}$ and $Q^{2}$, one can increase the number of hadrons included in the final-state jets (note that we do not count the beam jet as a final-state jet) by decreasing the value of $E_{t}$.

\section{D.D Factorized structure of jet cross sections}

Cross sections for the production of jets defined according to above $k_{\perp}$-algorithm have very simple factorization properties when computed in QCD perturbation theory. These factorization properties are discussed below. Some results of QCD calculations for jet rates are presented in Sec. 4 and a more detailed analysis will be presented elsewhere [12]. 
Let us consider the differential cross section $d \sigma^{n-j e t s}$ for $n$-jet production via off-shell photon exchange (the generalization to $W, Z$ exchange is straightforward)

$$
\begin{aligned}
d \sigma^{n-j e t s}\left(y, x, Q^{2} ; E_{t}^{2}, y_{\text {cut }}\right)= & \frac{4 \pi \alpha^{2}}{Q^{2}} \frac{d Q^{2}}{Q^{2}} \frac{d y}{y} \Theta\left(Q^{2}-\frac{y^{2} m^{2}}{1-y}\right) \\
& \cdot\left[(1-y) F_{2}^{(n)}+\frac{1}{2} y^{2} F_{1}^{(n)}\right] .
\end{aligned}
$$

Here $m$ is the initial-state lepton mass and $F_{i}^{(n)}(i=1,2)$ are the generalizations to exclusive $n$-jet production of the usual inclusive structure functions $F_{i}\left(x, Q^{2}\right)$. In particular we have

$$
F_{2}\left(x, Q^{2}\right)=\sum_{n=1}^{\infty} F_{2}^{(n)}, 2 x F_{1}\left(x, Q^{2}\right)=\sum_{n=1}^{\infty} F_{1}^{(n)} .
$$

The $n$-jet structure functions $F_{i}^{(n)}$ have remarkable properties [12] in QCD perturbation theory. They are independent of $y$ and have the factorizing structure

$$
\begin{aligned}
F_{i}^{(n)}\left(x, Q^{2} ; E_{t}^{2}, y_{\text {cut }}\right)= & \sum_{a} \int_{x}^{1} \frac{d z}{z} F_{a / h}\left(x / z, \mu_{F}^{2}\right) \\
& \cdot R_{i, a}^{(n)}\left(z, E_{t}^{2} / \mu_{F}^{2} ; \alpha_{S}\left(\mu_{R}^{2}\right), E_{t}^{2} / \mu_{R}^{2}, Q^{2} / E_{t}^{2}, y_{\text {cut }}\right) .
\end{aligned}
$$

Here $F_{a / h}$ are the universal parton distributions of the incoming hadron (the label $a$ denotes the parton type $a=q, \bar{q}, g$ ) evaluated at the factorization scale $\mu_{F}^{2}$. The jet-rate coefficient functions $R_{i, a}^{(n)}$ can be computed in QCD perturbation theory as power series expansions in $\alpha_{S}\left(\mu_{R}^{2}\right), \mu_{R}^{2}$ being the renormalization scale. In actual calculations, in order to have good convergence of the perturbative expansion for $R_{i, a}^{(n)}$ (i.e. to avoid large logarithmic terms $\left.\ln E_{t}^{2} / \mu_{F}^{2}, \ln E_{t}^{2} / \mu_{R}^{2}\right)$, one should choose $\mu_{F}^{2}, \mu_{R}^{2} \sim E_{t}^{2}$.

We stress that the formula (11) not only implies that initial-state collinear divergences can be absorbed in the universal parton densities, but also that the jet-rate coefficient functions do not depend explicitly on the DIS kinematic variables $x$ and $y$. This latter property is not valid if the $k_{\perp}$-clustering procedure is performed in a frame different from the Breit frame (apart from frames obtained from the Breit frame by finite $\left(x, y, Q^{2}\right)$-independent Lorentz transformations) and is not shared by invariant-mass JADE-type algorithms for DIS [13].

Obviously, after having defined jets according to the $k_{\perp}$-algorithm, one can measure and compute not only jet rates but also jet distributions depending on the jet momenta $\left\{p_{J}\right\}$. The corresponding factorization formula generalizes Eq. (11), with both $F_{i}^{(n)}$ and $R_{i, a}^{(n)}$ having explicit $p_{J}$-dependence.

\subsection{Jets in photoproduction}

The jet algorithm defined in Sec. 2.1 applies for $E_{t}^{2} \leq Q^{2}$ in the deep inelastic region of large $Q^{2}$. When $Q^{2}$ decreases below the hadron scale, $Q^{2} \leq 1 \mathrm{GeV}^{2}$, the process approaches the photoproduction limit. In this case one has to modify the clustering procedure slightly in order to take account of the hadronic component of the photon.

The $k_{\perp}$-clustering algorithm for photoproduction is defined in the centre-of-mass frame of the colliding photon and hadron. The pre-clustering procedure is the same as in Sec. 2.1 
except that in the steps $\left.a_{2}\right), a_{3}$ ) one has to compute also the transverse momentum $y_{k \gamma}$ relative to the incoming photon

$$
y_{k \gamma}=\frac{2\left(1-\cos \theta_{k \gamma}\right)}{E_{t}^{2}} E_{k}^{2},
$$

and take it into account in assigning particles to the "photon beam jet'. After the preclustering has been performed, jets are resolved as in Sec. 2.1. Obviously the hard scattering scale $E_{t}$ has to be smaller than the centre-of-mass energy $\sqrt{S}$.

The jet cross sections defined in this way satisfy the factorization formula

$$
\begin{aligned}
\sigma_{\gamma p}^{n-j e t s}\left(S, E_{t}^{2}, y_{\text {cut }},\left\{p_{J}\right\}\right)= & \sum_{a b} \int_{0}^{1} d z_{1} \int_{0}^{1} d z_{2} F_{a / \gamma}\left(z_{1}, E_{t}^{2}\right) F_{b / h}\left(z_{2}, E_{t}^{2}\right) \\
& \cdot \hat{\sigma}_{a b}^{(n)}\left(\frac{E_{t}}{z_{1} \sqrt{S}}, \frac{E_{t}}{z_{2} \sqrt{S}} ; \alpha_{S}\left(E_{t}^{2}\right), E_{t}^{2},\left\{p_{J}\right\}\right)
\end{aligned}
$$

where $F_{a / \gamma}$ are the parton distributions of the incoming photon and, as discussed after Eq. (11), we have chosen $\mu_{R}^{2}=\mu_{F}^{2}=E_{t}^{2}$.

\section{Provoking the $p \bar{p}$-collider community}

Jets in $p \bar{p}$-collisions are usually defined in terms of energy measured inside cones in pseudorapidity-azimuth space [14,15]. A lot of effort has recently been devoted to setting up a standard jet definition. An agreed cone definition was achieved at the 1990 Snowmass Workshop and is known as the "Snowmass Accord" [16].

As remarked earlier, jet definitions in terms of cones suffer from some disadvantages. Firstly, it is not straightforward to generalize this type of definition to multijet events because of ambiguities related to overlapping cones. Secondly, fixed-size cone jets cannot properly account for the coherence properties of QCD radiation. The underlying, uncorrelated event becomes mixed up with the coherent soft gluon radiation accompanying hard parton scattering, leading to phenomena like the 'pedestal effect' $[17,18]$. For these reasons, and in order to compare equivalent jet samples (with the same jet definition) from $e^{+} e^{-}$-annihilation, DIS and hadron-hadron collisions, we invite both experimentalists and theorists to consider the $k_{\perp}$-algorithm for $p \bar{p}$-collisions.

The definition of the $k_{\perp}$-algorithm for $p \bar{p}$-collisions is the same as that for photon-hadron collisions in Sec. 2.3. In the $p \bar{p}$ centre-of-mass frame one computes

$$
\begin{aligned}
& y_{k l}=\frac{2\left(1-\cos \theta_{k l}\right)}{E_{t}^{2}} \min \left(E_{k}^{2}, E_{l}^{2}\right), \\
& y_{k p}=\frac{2\left(1-\cos \theta_{k p}\right)}{E_{t}^{2}} E_{k}^{2}, \\
& y_{k \bar{p}}=\frac{2\left(1-\cos \theta_{k \bar{p}}\right)}{E_{t}^{2}} E_{k}^{2},
\end{aligned}
$$

then proceeds to pre-cluster final-state hadrons into beam jets and macro-jets, and finally resolves jets as for DIS in Sec. 2.1. In actual experiments, the same definition can be used replacing the energies and angles of the final-state hadron momenta by the corresponding quantities for calorimeter cells $k$. 
Jet cross sections computed according to the $k_{\perp}$-algorithm satisfy the factorization formula (13) with the replacement $\gamma \rightarrow \bar{p}$.

The $k_{\perp}$-clustering procedure can be generalized in a straightforward way to processes like $p \bar{p} \rightarrow Z(W)+$ jets.

\section{Some theoretical results}

In this Section we present some QCD calculations of jet cross sections in DIS.

The $n$-jet coefficient functions $R_{i, a}^{(n)}$ in Eq. (11) are calculable in QCD perturbation theory and the tools already exist for a complete two-loop calculation [19]. However, these coefficient functions depend on several kinematical scales $\left(x, Q^{2} / E_{t}^{2}, y_{\text {cut }}\right)$, and their perturbative expansion in $\alpha_{S}$ is reliable only as long as these scales are not very different from unity. In the kinematic regimes $x \rightarrow 1,0, Q^{2} \gg E_{t}^{2}, y_{\text {cut }} \ll 1$ the perturbative expansion has large logarithmic terms of the type $\alpha_{S}^{n} L^{m}(m \leq 2 n)$ where $L=\ln (1-x)$, $\ln x, \ln Q^{2} / E_{t}^{2}$ and/or $\ln y_{\text {cut }}$. These logarithmic contributions spoil the convergence of the perturbative series in $\alpha_{S}$ and have to be resummed to all orders. In the following we consider only the case of moderate values of $x(x, 1-x=O(1))$ and concentrate on the resummation of the large logarithms $\ln Q^{2} / E_{t}^{2}$ and $\ln y_{\text {cut }}$. In particular we present the results of an all-order calculation of the terms $\alpha_{S}^{n}\left(\ln Q^{2} / E_{t}^{2}\right)^{p}\left(\ln y_{\text {cut }}\right)^{q}$ with next-to-leading logarithmic accuracy $(p+q=2 n, 2 n-1)$.

To next-to-leading logarithmic order the $n$-jet structure functions satisfy the generalized Callan-Gross relation $F_{2}^{(n)}=F_{1}^{(n)}$ (i.e. the $n$-jet longitudinal structure functions vanish). Therefore, from now on, we shall drop the photon polarization index $i$ in $F_{i}^{(n)}$. Moreover, as discussed in Sec. 2.2, we shall fix the factorization and renormalization scales at the physical value $\mu_{R}^{2}=\mu_{F}^{2}=E_{t}^{2}$.

Let us introduce the DIS generating function $\left(Q_{0}=E_{t} \sqrt{y}_{\text {cut }}\right)$

$$
\phi_{D I S}\left(x, Q ; E_{t}, Q_{0}, u\right) \equiv \sum_{n=1}^{\infty} F^{(n)}\left(x, Q^{2} ; E_{t}^{2}, y_{\text {cut }}\right) u^{n}
$$

which gives the $n$-jet structure functions $F^{(n)}$ by successive differentiation at $u=0$. Since, according to Eq. (11), the jet structure functions are obtained by convoluting jet coefficient functions and parton distributions, it is convenient to consider the $N$-moments

$$
\phi_{N, D I S}\left(Q ; E_{t}, Q_{0}, u\right) \equiv \int_{0}^{1} d x x^{N-1} \phi_{D I S}\left(x, Q ; E_{t}, Q_{0}, u\right),
$$

and the analogous moments of $F^{(n)}$ and $R^{(n)}$.

The DIS generating function for jets defined according to the $k_{\perp}$-algorithm can be computed to next-to-leading logarithmic accuracy using the coherent branching formalism $[20,21]$. We find [12]

$$
\phi_{N, D I S}\left(Q ; E_{t}, Q_{0}, u\right)=\sum_{q} e_{q}^{2}\left[\mathcal{Z}_{N, q}\left(Q ; E_{t}, Q_{0}, u\right)+\mathcal{Z}_{N, \bar{q}}\left(Q ; E_{t}, Q_{0}, u\right)\right] \phi_{q}\left(Q, Q_{0}, u\right),
$$

where $\phi_{q}$ is the time-like generating function of the struck quark and $\mathcal{Z}_{q}$ is the space-like generating function of the incoming hadron. 
The time-like generating functions $\phi_{a}(a=q, \bar{q}, g)$ satisfy the equations [2]

$$
\begin{gathered}
\phi_{q}\left(Q, Q_{0}, u\right)=u \exp \left(\int_{Q_{0}}^{Q} d q \Gamma_{q}(Q, q)\left[\phi_{g}\left(q, Q_{0}, u\right)-1\right]\right), \\
\phi_{g}\left(Q, Q_{0}, u\right)=u \exp \left(\int_{Q_{0}}^{Q} d q\left\{\Gamma_{g}(Q, q)\left[\phi_{g}\left(q, Q_{0}, u\right)-1\right]-\Gamma_{f}(q)\right\}\right) \\
\cdot\left\{1+u \int_{Q_{0}}^{Q} d q \Gamma_{f}(q) \exp \left(\int _ { Q _ { 0 } } ^ { q } d q ^ { \prime } \left\{\left[2 \Gamma_{q}\left(q, q^{\prime}\right)-\Gamma_{g}\left(q, q^{\prime}\right)\right]\right.\right.\right. \\
\text { - } \left.\left.\left.\left[\phi_{g}\left(q^{\prime}, Q_{0}, u\right)-1\right]+\Gamma_{f}\left(q^{\prime}\right)\right\}\right)\right\},
\end{gathered}
$$

where we have introduced the following parton splitting probabilities

$$
\begin{gathered}
\Gamma_{q}(Q, q)=\frac{2 C_{F}}{\pi} \frac{\alpha_{S}(q)}{q}\left(\log \frac{Q}{q}-\frac{3}{4}\right), \\
\Gamma_{g}(Q, q)=\frac{2 C_{A}}{\pi} \frac{\alpha_{S}(q)}{q}\left(\log \frac{Q}{q}-\frac{11}{12}\right), \\
\Gamma_{f}(q)=\frac{N_{f}}{3 \pi} \frac{\alpha_{S}(q)}{q},
\end{gathered}
$$

in next-to-leading logarithmic approximation.

The space-like evolution of jets is taken into account by the generating functions $\mathcal{Z}_{a}$ which are obtained by solving an evolution equation of jet calculus type [12]

$$
\begin{aligned}
& \mathcal{Z}_{N, a}\left(Q ; E_{t}, Q_{0}, u\right)=\tilde{\phi}_{a}\left(Q ; E_{t}, Q_{0}, u\right)\left\{F_{N, a}\left(E_{t}^{2}\right)\right. \\
& \left.+\sum_{b} \int_{E_{t}}^{Q} \frac{d q}{q} \frac{\alpha_{S}(q)}{\pi} \mathcal{Z}_{N, b}\left(q ; E_{t}, Q_{0}, u\right) \gamma_{N, b a(c)} \phi_{c}\left(q, Q_{0}, u\right)\left[\tilde{\phi}_{a}\left(q ; E_{t}, Q_{0}, u\right)\right]^{-1}\right\} .
\end{aligned}
$$

Here $F_{N, a}\left(E_{t}^{2}\right)$ are the $N$-moments of the parton distributions, $\gamma_{N, b a(c)}$ are the one-loop anomalous dimensions for the parton splitting $b \rightarrow a$ ( $c$ is the parton emitted in the splitting process), $\phi_{c}$ are the time-like generating functions in Eqs. (18), (19) and the twoscale generating functions $\tilde{\phi}_{a}$ are given by

$$
\begin{gathered}
\tilde{\phi}_{q}\left(Q ; E_{t}, Q_{0}, u\right)=\exp \left(\int_{E_{t}}^{Q} d q \Gamma_{q}(Q, q)\left[\phi_{g}\left(q, Q_{0}, u\right)-1\right]\right), \\
\tilde{\phi}_{g}\left(Q ; E_{t}, Q_{0}, u\right)=\exp \left(\int_{E_{t}}^{Q} d q\left(\Gamma_{g}(Q, q)+\Gamma_{f}(q)\right)\left[\phi_{g}\left(q, Q_{0}, u\right)-1\right]\right) .
\end{gathered}
$$

Eq. (23) has a simple solution if one considers the flavour non-singlet generating function

$$
\mathcal{Z}_{N, N S}\left(Q ; E_{t}, Q_{0}, u\right)=\mathcal{Z}_{N, q}\left(Q ; E_{t}, Q_{0}, u\right)-\mathcal{Z}_{N, \bar{q}}\left(Q ; E_{t}, Q_{0}, u\right)
$$

Using Eqs. (23-25) we find

$$
\mathcal{Z}_{N, N S}\left(Q ; E_{t}, Q_{0}, u\right)=F_{N, N S}\left(E_{t}^{2}\right) \tilde{\phi}_{q}\left(Q, E_{t}, Q_{0}, u\right) \exp \left[\int_{E_{t}}^{Q} \frac{d q}{q} \gamma_{N, N S} \frac{\alpha_{S}(q)}{\pi} \phi_{g}\left(q, Q_{0}, u\right)\right] \text {. }
$$


This result shows explicitly that the structure function $F_{N}\left(E_{t}^{2}\right)$ factorizes with respect to the jet cross section coefficient functions.

Eq. (27) has a simple physical interpretation. Introducing the Sudakov form factors

$$
\begin{aligned}
& \Delta_{q}\left(Q, Q_{0}\right)=\exp \left(-\int_{Q_{0}}^{Q} d q \Gamma_{q}(Q, q)\right), \\
& \Delta_{g}\left(Q, Q_{0}\right)=\exp \left(-\int_{Q_{0}}^{Q} d q\left[\Gamma_{g}(Q, q)+\Gamma_{f}(q)\right]\right), \\
& \Delta_{f}\left(Q, Q_{0}\right)=\left[\Delta_{q}\left(Q, Q_{0}\right)\right]^{2} / \Delta_{g}\left(Q, Q_{0}\right),
\end{aligned}
$$

we can rewrite Eq. (27) as follows

$$
\begin{aligned}
\mathcal{Z}_{N, N S}\left(Q ; E_{t}, Q_{0}, u\right) & =F_{N, N S}\left(E_{t}^{2}\right) \Delta_{q}\left(Q, E_{t}\right) \\
& \cdot \exp \left[\int_{E_{t}}^{Q} \frac{d q}{q} \frac{\alpha_{S}(q)}{\pi}\left(2 C_{F} \ln \frac{Q}{q}+\gamma_{N, N S}-\frac{3}{2} C_{F}\right) \phi_{g}\left(q, Q_{0}, u\right)\right] .
\end{aligned}
$$

The exponential factor on the right-hand side represents multiple jet production from the space-like quark exchanged in the $t$-channel. More precisely, the term proportional to $\left(\gamma_{N, N S}-\frac{3}{2} C_{F}\right)$ is due to hard gluon radiation at large $p_{\perp}$, whilst the term proportional to $2 C_{F} \ln Q / q$ is due to soft gluon radiation at angles $\theta$ larger than the natural opening angle $\theta_{t}=E_{t} / Q$ of the beam jet. Finally, the $\Delta_{q}$ term is the Sudakov form factor which gives the probability that the quark propagates in the $t$-channel without producing any macro-jets with $k_{\perp}>E_{t}$.

The result (27) has a straightforward generalization for the parton generating functions $\mathcal{Z}_{N, a}$. In this case the non-singlet anomalous dimension is replaced by the anomalous dimension matrix $\gamma_{N, b a(c)}$ and the exponential factor is path-ordered.

We conclude this section by writing explicitly the first few DIS jet structure functions for the simple case that $E_{t}=Q$. From Eqs. (17), (23) we see that in this case only the struck quark contributes to jet production. To next-to-leading logarithmic accuracy in $\ln y_{\text {cut }}$, we find by using Eqs. (18), (19)

$$
\begin{aligned}
F^{(1)}\left(x, Q^{2} ; E_{t}^{2}=Q^{2}, y_{\text {cut }}\right) & =F_{2}\left(x, Q^{2}\right) \Delta_{q}\left(Q, Q_{0}\right) \\
F^{(2)}\left(x, Q^{2} ; E_{t}^{2}=Q^{2}, y_{\text {cut }}\right) & =F_{2}\left(x, Q^{2}\right) \Delta_{q}\left(Q, Q_{0}\right) \int_{Q_{0}}^{Q} d q \Gamma_{q}(Q, q) \Delta_{g}\left(q, Q_{0}\right), \\
F^{(3)}\left(x, Q^{2} ; E_{t}^{2}=Q^{2}, y_{\text {cut }}\right) & =F_{2}\left(x, Q^{2}\right) \Delta_{q}\left(Q, Q_{0}\right),\left\{\frac{1}{2}\left[\int_{Q_{0}}^{Q} d q \Gamma_{q}(Q, q) \Delta_{g}\left(q, Q_{0}\right)\right]^{2}\right. \\
& +\int_{Q_{0}}^{Q} d q \Gamma_{q}(Q, q) \Delta_{g}\left(q, Q_{0}\right) \int_{Q_{0}}^{q} d q^{\prime} \Gamma_{g}\left(q, q^{\prime}\right) \Delta_{g}\left(q^{\prime}, Q_{0}\right) \\
& \left.+\int_{Q_{0}}^{Q} d q \Gamma_{q}(Q, q) \Delta_{g}\left(q, Q_{0}\right) \int_{Q_{0}}^{q} d q^{\prime} \Gamma_{f}\left(q^{\prime}\right) \Delta_{f}\left(q^{\prime}, Q_{0}\right)\right\}
\end{aligned}
$$

where $F_{2}$ is the usual (inclusive) DIS structure function.

The derivation of the results presented in this section, together with more QCD calculations, will be given elsewhere [12]. 


\section{Discussion}

Our main aim in the work presented here has been to extend to other processes the $k_{\perp}$-algorithm originally proposed for $e^{+} e^{-}$annihilation. An essentially new feature appears whenever there are hadrons in the initial state, namely the 'beam jet(s)' containing initialstate QCD radiation from the incoming coloured partons of the hard scattering process. The requirements that the beam jets should be insensitive to hadron remnants and the soft underlying event, and that their definition should permit the universal factorization of collinear singularities, lead naturally to the two-step clustering procedure defined in Sect. 2.1. First the beam jet(s) and outgoing 'macro-jets' are assembled at some hardscattering resolution scale $E_{t}$, and then the multi-jet substructure of the macro-jets is explored using a finer resolution $\sqrt{y_{\text {cut }}} E_{t}$, always using the test variables of Eqs. (7),(8) as the criterion of resolvability.

Our most important general results on multijet cross sections, defined in the way we have proposed, are Eqs. (11) for DIS and (13) for photon-hadron collisions and the generalization of the latter to hadron-hadron scattering. Eq. (11) shows explicitly the good factorization properties which permit the definition of universal, perturbatively computable multijet coefficient functions $R_{i, a}^{(n)}$ which have no intrinsic $x$ - or $y$-dependence and have only to be convoluted with the ordinary parton distributions $F_{a / h}$ in order to predict measurable cross sections.

Since the $k_{\perp}$-clustering procedure is not explicitly Lorentz-invariant the frame of reference in which it is performed has to be specified. For deep inelastic scattering the Breit frame is preferred, while in collisions between hadrons it is the hadron-hadron centre-ofmass frame. Only in these frame is intrinsic dependence on $x$, beam energy, and for DIS $y$, absent from the multijet fractions.

The formulae presented in Eqs. (32-34) for the multijet cross sections in DIS are examples of the results that can be obtained in the next-to-leading logarithmic approximation by means of the jet calculus approach. For more precise predictions, in particular to measure $\alpha_{S}$ by comparing cross sections for different numbers of jets, a full fixed-order perturbative calculation using the $k_{\perp}$-algorithm should be performed. One could then for example insert the measured $m$ - and $n$-jet DIS structure functions directly into the relation $(m>n)$

$$
\begin{aligned}
F_{i}^{(m)}\left(x, Q^{2} ; E_{t}^{2}, y_{\text {cut }}\right)= & \sum_{j} \int_{x}^{1} \frac{d z}{z} F_{j}^{(n)}\left(z, Q^{2} ; E_{t}^{2}, y_{\text {cut }}\right) \\
& \cdot \mathcal{R}_{i j}^{(m, n)}\left(x / z ; \alpha_{S}\left(\mu_{R}^{2}\right), E_{t}^{2} / \mu_{R}^{2}, Q^{2} / E_{t}^{2}, y_{\text {cut }}\right) .
\end{aligned}
$$

where the jet rate matrix $\mathcal{R}_{i j}^{(m, n)}$ is computable in perturbation theory, and measure $\alpha_{S}$ (including its running, by setting $\mu_{R}=E_{t}$ and varying $\left.E_{t}\right)$. Notice that the inclusive structure functions $F_{i}\left(x, \mu_{F}^{2}\right)$ and the corresponding parton densities are not involved in Eq. (35) and in particular there is no factorization scale ambiguity. In fact, the jet-rate matrix $\mathcal{R}_{i j}^{(m, n)}$ does not depend on the factorization scheme for initial-state collinear singularities.

The $k_{\perp}$-algorithm proposed in this paper allows one to select similar jet samples in different hard processes. It is a clustering algorithm which is defined at a fully exclusive level and thus can be applied to study jet-rates as well as jet-distributions. Its use can help in understanding and testing the universality properties of QCD. 


\section{Acknowledgements}

We would like to acknowledge helpful discussions with Zoltan Kunszt and Karlheinz Meier.

\section{References}

1. G. Hanson et al., Phys. Rev. Lett. 35 (1975) 1609.

2. S. Catani, Yu.L. Dokshitzer, M. Olsson, G. Turnock and B.R. Webber, Phys. Lett. 269B (1991) 432.

3. S. Catani, Yu.L. Dokshitzer, M. Olsson, G. Turnock and B.R. Webber, Cambridge preprint Cavendish-HEP-91/12, in preparation.

4. S. Catani, preprint CERN-TH.6281/91, to be published in Proc. 17th Workshop of the INFN Eloisatron Project, Erice, June, 1991.

5. N. Brown and W.J. Stirling, Phys. Lett. 252B (1990) 657; Zeit. Phys. C53 (1992) 629.

6. S. Bethke, Z. Kunszt, D.E. Soper and W.J. Stirling, Nucl. Phys. B370 (1992) 310.

7. S. Catani, Yu.L. Dokshitzer, F. Fiorani and B.R. Webber, preprint CERN-TH.6328/91, to be published in Nucl. Phys. B; preprint CERN-TH.6419/92.

8. B. Flaugher and K. Meier, FERMILAB-Conf.-90/248-E.

9. JADE Collaboration, W. Bartel et al., Zeit. Phys. C33 (1986) 23; S. Bethke et al., Phys. Lett. 213B (1988) 235.

10. G. Sterman and S. Weinberg, Phys. Rev. Lett. 39 (1977) 1436.

11. M. Fleischer, G. Ingelman, G. Knies, K. Meier, C. Pichler and A. Wegner, contribution to Proc. Workshop on Physics at HERA, DESY, Hamburg, October 1991.

12. S. Catani, Yu.L. Dokshitzer and B.R. Webber, in preparation.

13. P.N. Burrows, G. Ingelman and E. Ros, Zeit. Phys. C39 (1988) 257; J.G. Korner, E. Mirkes and G.A. Schuler, Int. J. Mod. Phys. A4 (1989) 1781; T. Brodkorb, J.G. Korner, E. Mirkes and G.A. Schuler, Zeit. Phys. C44 (1989) 415.

14. UA1 Collaboration, G. Arnison et al., Phys. Lett. 132B (1983) 214; CDF Collaboration, F. Abe et al., Phys. Rev. Lett. 62 (1988) 613.

15. S.D. Ellis, Z. Kunszt and D.E. Soper, Phys. Rev. Lett. 64 (1990) 2121, Phys. Rev. D 40 (1989) 2188; F. Aversa, M. Greco, P. Chiappetta and J.Ph. Guillet, Phys. Rev. Lett. 65 (1990) 401, Zeit. Phys. C49 (1991) 459.

16. J.E. Huth et al., FERMILAB-Conf.-90/249-E.

17. UA1 Collaboration, C. Albajar et al., Nucl. Phys. B309 (1988) 405. 
18. G. Marchesini and B.R. Webber, Phys. Rev. D 38 (1988) 3419.

19. R.K. Ellis, D.A. Ross and A.E. Terrano, Nucl. Phys. B178 (1981) 421; E.B. Zijlstra and W.L. van Neerven, preprint INLO-PUB-1/92.

20. Yu.L. Dokshitzer, V.A. Khoze, A.H. Mueller and S.I. Troyan, Basics of Perturbative QCD, Editions Frontieres, Paris, 1991.

21. S. Catani, G. Marchesini and B.R. Webber, Nucl. Phys. B349 (1991) 635. 